\title{
La qualité du lait chauffé à ultra-haute température comparée à celle du lait pasteurisé et stérilisé dans la bouteille
}

\author{
par \\ J. MOTTAR et M. NAUDTS \\ Station Laitière de l'Etat, Melle \\ Centre de Recherches Agronomiques de l'Etat, \\ Gand (Belgique)
}

\section{INTRODUCTION}

En élaborant la technique du réchauffement à ultra-haute température du lait on a visé à obtenir, après le soutirage aseptique, un lait à identité propre et qui ne présenterait pas les inconvénients du lait stérilisé dans la bouteille. On a cherché a réunir la qualité organoleptique et nutritionnelle du lait pasteurisé et la conservabilité du lait stérilisé par la méthode traditionnelle.

Selon la Fédération Internationale de Laiterie, le lait UHT doit devenir trouble quand on le soumet au test d'Aschaffenburg (IDF, 1972). Une étude de la qualité du lait UHT produit en Belgique, faite en 1978 aux fins d'orientation (Mottar et Naudts, 1979) a révélé que l'effet de réchauffement de certains appareils de traitement UHT indirect était si intense que, tout comme pour le lait stérilisé dans la bouteille, le test de turbidité donnait un résultat négatif. De ce fait, le lait UHT risque de perdre son caractère propre.

Cette constatation nous a conduit à rechercher en quelle mesure le lait UHT vendu sur le marché belge présente encore un caractère propre, sous le rapport organoleptique et nutritionnel, comparativement au lait pasteurisé et stérilisé dans la bouteille par la méthode traditionnelle. Nous avons recherché en outre, à l'aide de quelques paramètres physico-chimiques étroitement liés à l'intensité de réchauffement, si des méthodes analytiques permettent de distinguer les différents types de lait de consommation. 


\section{MATERIEL ET METHODES}

\section{II.1. Echantillonnage}

Des échantillons de lait ont été prélevés aussitôt après la production dans six laiteries. On a échantillonné ainsi, une à trois fois, du lait de trois installations de pasteurisation, de quatre unités de stérilisation en bouteille et de onze appareils UHT, dont cinq à système direct et six à système indirect. Il s'agissait uniquement de lait entier (3,5 p. 100 de matière grasse).

\section{II.2. Appréciation du goût}

Les goûts des différents types de lait de consommation ont été comparés par recours au test du triangle. A cette fin il a été fait appel à une équipe d'experts de la Station Laitière de l'Etat, composée de dix personnes. Le pourcentage d'experts qui pouvaient faire la distinction exacte a été noté.

Le lait UHT a été expertisé après $10 \mathrm{j}$ et après 4 semaines de conservation à $1^{\circ} \mathrm{C}$ et comparé avec du lait fraîchement pasteurisé. Il a été comparé d'autre part avec du lait stérilisé dans la bouteille, après 2 semaines de conservation à $1^{\circ} \mathrm{C}$ des deux types de lait.

\section{II.3. Méthodes d'analyse}

La lysine disponible a été déterminée par la méthode de Carpenter modifiée, telle que décrite par Booth (1971).

Le dosage des vitamines hydrosolubles $\mathrm{B}_{12}$ et l'acide folique a été effectué selon la méthode microbiologique, prescrite par l' " Association of Official Analytical Chemists » (1975). Les micro-organismes utilisés pour les tests étaient, respectivement, Lactobacillus leichmanii ATCC 7830 et Streptococcus faecalis ATCC 8043. La méthode de titration a été appliquée pour le calcul de la teneur en vitamines.

La teneur en acide ascorbique a été déterminée par titrimétrie au dichlorophénol-indophénol (Hanson et Olsson, 1974).

Les fractions azotées ont été déterminées par la technique d'Aschaffenburg et Drewey (1959). L'azote a été dosé par micro-Kjeldahl. La teneur en azote des protéines lactosériques (WPN) a été calculée d'après la différence entre l'azote non caséique (NCN) et l'azote non protéique (NPN).

L'indice WPN a été déterminé d'après le test de Harland-Ashworth (Kuramoto et al., 1959). Les résultats ont été exprimés en valeurs de transmission, mesurées à $420 \mathrm{~nm}$.

La méthode IDF a été suivie pour l'application des tests de turbidité (1972). La turbidité de $20 \mathrm{ml}$ de filtrat chauffé a toutefois été mesurée au moyen d'un néphélomètre Fisher DRT-100, après 
refroidissement à la température de chambre. Les résultats sont exprimés en unités de turbidité néphélométrique (NTU).

La détermination de l'hydroxyméthylfurfural (HMF) est basée sur la méthode de Keeney et Basette (1959). Pour le calcul de la concentration $\mathrm{Y}$ de l'HMF ( $\mu \mathrm{mol} / \mathrm{l})$ nous avons employé l'équation de régression $\mathrm{Y}=87,5 \mathrm{X}-4,8(\mathrm{X}=$ densité optique mesurée à $443 \mathrm{~nm})$.

Les analyses ont été faites aussi rapidement que possible après la production, au plus tard après 2 semaines de conservation à $1^{\circ} \mathrm{C}$. La vitamine $\mathrm{C}$ a toujours été déterminée le jour même de la production.

\section{RESULTATS ET COMMENTAIRE}

\section{III.1. Aspects organoleptiques}

Un des principaux critères de qualité d'une denrée alimentaire est sa qualité organoleptique dont le goût est l'élément le plus important. Puisque le procédé UHT a été élaboré en vue de l'obtention d'un produit à propriétés organoleptiques analogues à celles du lait pasteurisé, nous avons comparé, par le test du triangle, le goût de laits UHT avec celui d'un lait fraîchement pasteurisé de bonne qualité. Le tableau 1 reproduit les résultats de cette comparaison. $10 \mathrm{j}$ après la production, un nombre significatif d'experts ont pu percevoir une différence de goût entre le lait qui avait été chauffé à ultra-haute

\section{TABLEAU 1}

Comparaison du goût du lait UHT et du lait pasteurisé

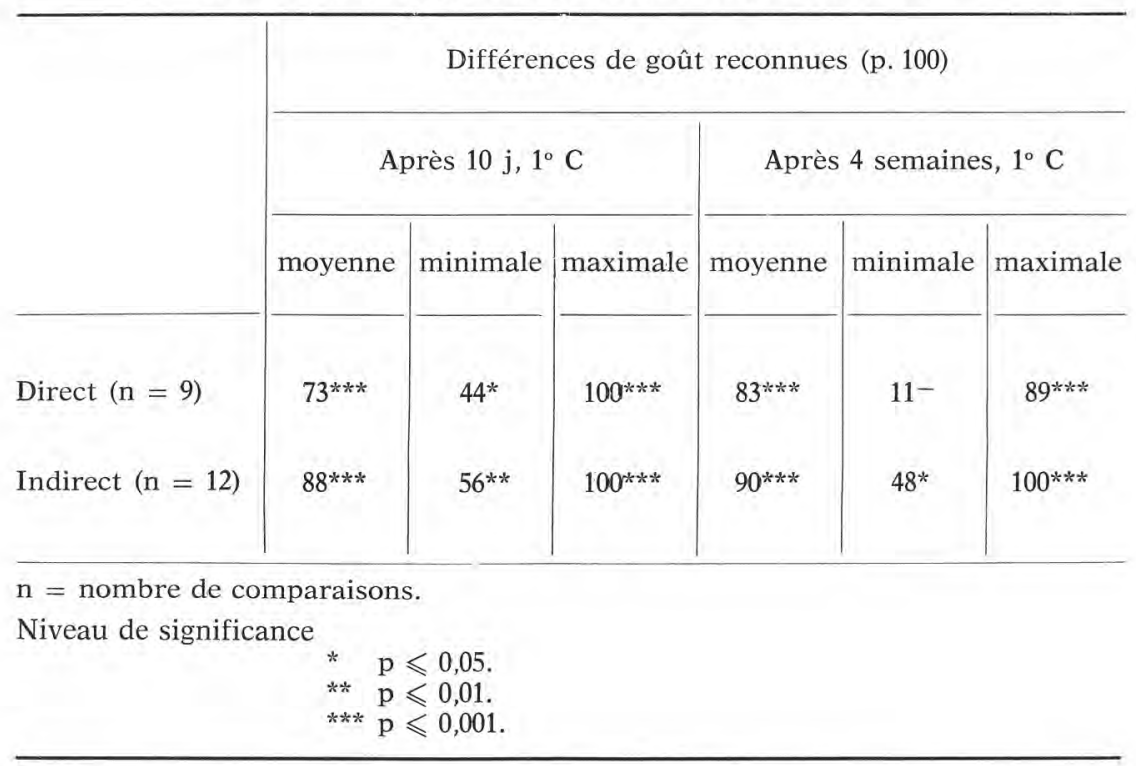


température et le lait pasteurisé. Le lait chauffé indirectement se distinguait plus nettement. Ces différences résidaient surtout en un goût de cuit plus ou moins prononcé. Ce goût s'atténuant avec le temps, les expertises ont été répétées après 4 semaines. Le lait UHT avait été conservé entre-temps à $1^{\circ} \mathrm{C}$. Les résultats étaient encore comparables avec les premiers.

Nous avons recherché de manière analogue en quelle mesure le goût du lait UHT se distingue de celui du lait stérilisé dans la bouteille. Les résultats sont présentés dans le tableau 2. Le lait chauffé directement a été distingué dans une mesure très significative du lait stérilisé par la méthode traditionnelle et cela quel que fût le conditionnement, en bouteilles de polyéthylène ou en bouteilles de verre. Les différences étaient en moyenne, beaucoup moins significatives quand le lait avait subi le traitement UHT indirect ; dans certains cas elles n'étaient même pas significatives.

TABLEAU 2

Comparaison du goût du lait UHT et du lait stérilisé en bouteille

\begin{tabular}{|c|c|c|c|}
\hline & \multicolumn{3}{|c|}{ Différences de goût reconnues (p. 100) } \\
\hline & moyenne & minimale & maximale \\
\hline $\begin{array}{l}\text { UHT, direct } \\
\text { stérilisé en polyéthylène } \\
(\mathrm{n}=20)\end{array}$ & $76^{* * *}$ & $56^{* * *}$ & $100^{* * *}$ \\
\hline $\begin{array}{l}\text { UHT, direct } \\
\text { stérilisé en verre } \\
(\mathrm{n}=12)\end{array}$ & $84^{* * *}$ & $75 * * *$ & $89 * * *$ \\
\hline $\begin{array}{l}\text { UHT, indirect } \\
\text { stérilisé en polyéthylène } \\
(\mathrm{n}=34)\end{array}$ & $48^{*}$ & $11^{-}$ & $89 * * *$ \\
\hline $\begin{array}{l}\text { UHT, indirect } \\
\text { stérilisé en verre } \\
(\mathrm{n}=16)\end{array}$ & $52^{* *}$ & $34^{-}$ & $67 * * *$ \\
\hline \multicolumn{4}{|c|}{$\mathrm{n}=$ nombre de comparaisons. } \\
\hline $\begin{aligned} \text { Niveau de significance } & \\
& * \\
& * * \\
& * * *\end{aligned}$ & \multicolumn{3}{|c|}{$\begin{array}{ll}* & p \leqslant 0,05 \\
* * & p \leqslant 0,01 \\
* * * & p \leqslant 0,001\end{array}$} \\
\hline
\end{tabular}




\section{III.2. Aspects nutritionnels}

Le lait est une source unique de composants nutritifs essentiels. Sa valeur nutritive résulte de sa teneur en composants chimiques et de la composition et de la structure de ceux-ci. Une modification de ces composants résultant d'un processus thermique peut modifier la valeur du lait sous le rapport de la physiologie de la nutrition.

La fraction lipidique n'est que peu ou pas influencée par un traitement thermique. Les acides gras importants pour la nutrition, en l'occurrence les acides gras inférieurs et les acides gras insaturés, ne sont pas modifiés. De plus, un traitement thermique normal n'entraîne pas de formation de produits de dégradation de la matière grasse, qui nuiraient à la valeur nutritive du lait.

La composition en minéraux ne subit que peu de modifications notables sous l'influence d'un processus thermique.

Les protéines du lait ont, de par leur nature, une grande valeur biologique, qu'elles doivent à leur teneur en acides aminés essentiels. Certains de ceux-ci peuvent être impliqués dans un processus induit par la chaleur. La lysine peut se lier à des sucres réducteurs par la réaction de Maillard et la cystine peut être impliquée dans certaines interréactions de protéines. Ceci fait diminuer la disponibilité d'acides aminés essentiels et, par conséquent, la valeur nutritive.

Le tableau 3 donne un aperçu des pertes moyennes en lysine disponible correspondant aux traitements thermiques du lait, dont il est question ici. Ces données montrent que les différents procédés

\section{TABLEAU 3}

Effet du traitement thermique sur la disponibilité de lysine (pertes en p. 100)

\begin{tabular}{lr|c|c|c|c}
\hline \multicolumn{1}{c|}{ Traitement thermique } & Moyenne & Minimale & Maximale & Déviat. stand. \\
& & & & & \\
& & & & \\
Pasteurisation & $(\mathrm{n}=9)$ & 2,0 & 0 & 5,8 & 1,8 \\
UHT, direct & $(\mathrm{n}=10)$ & 4,3 & 1,4 & 7,7 & 2,4 \\
UHT, indirect & $(\mathrm{n}=18)$ & 6,5 & 1,9 & 19,9 & 6,0 \\
Stérilisation en PE & $(\mathrm{n}=12)$ & 8,9 & 6,0 & 24,5 & 6,2 \\
Stérilisation en verre & $(\mathrm{n}=9)$ & 11,3 & 8,1 & 26,1 & 6,5 \\
& & & & & \\
\hline
\end{tabular}

$\mathbf{n}=$ nombre d'échantillons. 
de production ont influé différemment sur les pertes en lysine. Dans le groupe des laits UHT on a relevé une différence entre le procédé direct et le procédé indirect. La disponibilité en lysine a diminué en moyenne de 6,5 p. 100 sous l'effet du mode de chauffage indirect, contre 4,3 p. 100 du fait du procédé direct. On a toutefois noté dans certains cas, pour le traitement indirect, des pertes qui approchent celles résultant d'une stérilisation en bouteille.

Les effets du réchauffement sur la valeur nutritionnelle du lait peuvent aussi être jugés d'après les pertes en vitamines. Les vitamines liposolubles A, D et $\mathrm{E}$ sont relativement thermostables, la riboflavine hydrosoluble $\left(\mathrm{B}_{2}\right)$ l'est également. D'autres vitamines hydrosolubles telles que la thiamine $\left(B_{1}\right)$, la pyridoxine $\left(B_{6}\right)$, la cyanocobalamine $\left(B_{12}\right)$, l'acide folique et la vitamine $C$ sont thermosensibles.

Le tableau 4 illustre l'influence des traitements thermiques sur les pertes en vitamine $B_{12}$, en acide folique et en vitamine $C$. Les

\section{TABLEAU 4}

Effet du traitement thermique sur quelques vitamines hydrosolubles (pertes en p. 100)

\begin{tabular}{lr|c|c|c}
\hline \multicolumn{1}{c|}{ Traitement thermique } & Vit. $\mathrm{B}_{12}$ & Acide folique & \multirow{2}{*}{ Vit. C } \\
\cline { 1 - 2 } & & & & \\
Pasteurisation & $(\mathrm{n}=7)$ & 4,6 & 7,3 & 12,8 \\
UHT, direct & $(\mathrm{n}=9)$ & 16,8 & 19,6 & 17,7 \\
UHT, indirect & $(\mathrm{n}=17)$ & 30,1 & 35,1 & 31,6 \\
Stérilisation en PE & $(\mathrm{n}=12)$ & 36,5 & 45,6 & 50,0 \\
Stérilisation en verre & $(\mathrm{n}=8)$ & 39,0 & 54,8 & 66,5 \\
\end{tabular}

$\mathrm{n}=$ nombre d'échantillons.

pertes provoquées par la pasteurisation étaient inférieures, en règle générale, à 13 p. 100. Ces pertes étaient plus élevées dans le lait UHT et l'on pouvait faire une distinction entre le système direct (17 à 20 p. 100) et le système indirect (30 à 35 p. 100). Dans ce dernier processus, les pertes en vitamines étaient comparables à celles résultant d'une stérilisation classique en bouteille de polyéthylène. 


\section{III.3. Aspects analytiques}

\section{III.3.1. Teneur en protéines lactosériques}

L'intensité de chaleur des processus thermiques a été étudiée par détermination du degré de dénaturation des protéines du sérum. Les protéines lactosériques dissoutes sont, en effet, très sensibles à la température. Le tableau 5 donne un aperçu des quantités restantes

\section{TABLEAU 5}

Les quantités d'azote de protéines lactosériques du lait de consommation (mg/100 g)

\begin{tabular}{ll|c|c|c|c}
\hline \multicolumn{1}{c|}{ Traitement thermique } & Moyenne & Minimale & Maximale & Déviat. stand. \\
& & & & & \\
& & & & \\
Lait cru & $(\mathrm{n}=26)$ & 95,5 & 71,2 & 110,8 & 6,5 \\
Pasteurisation & $(\mathrm{n}=8)$ & 80,8 & 70,8 & 93,1 & 6,0 \\
UHT, direct & $(\mathrm{n}=11)$ & 38,8 & 31,6 & 47,7 & 5,0 \\
UHT, indirect & $(\mathrm{n}=15)$ & 27,6 & 22,1 & 35,7 & 4,0 \\
Stérilisation en PE & $(\mathrm{n}=12)$ & 20,5 & 16,0 & 22,9 & 3,9 \\
Stérilisation en verre & $(\mathrm{n}=9)$ & 21,9 & 15,9 & 25,5 & 3,1 \\
& & & & & \\
\hline
\end{tabular}

$\mathrm{n}=$ nombre d'échantillons.

d'azote de protéines lactosériques des différentes catégories de lait de consommation. Les données montrent que les divers processus thermiques donnent des charges thermiques très différentes.

La pasteurisation n'a influé que dans une faible mesure sur les protéines du sérum. La diminution de protéines natives a été de 15 p. 100 en moyenne. L'effet du réchauffement à ultra-haute température a été nettement plus grand (50 à 77 p. 100). Frappante est ici l'importante différence moyenne entre les systèmes direct et indirect. L'intensité de réchauffement de certains appareils indirects était comparable à celle d'une stérilisation traditionnelle dans la bouteille. 
De telles différences de charge thermique entre les différents procédés de production de lait de consommation peuvent expliquer les différences de qualité observées.

La détermination de la teneur restante en azote de protéines lactosériques permet de faire une différence bien nette entre les différents types de lait de consommation. La méthode micro-Kjeldahl est toutefois beaucoup trop laborieuse pour les analyses de routine. C'est pourquoi nous avons examiné si la détermination rapide de l'indice WPN (WPNI) permet de faire une distinction. Les résultats sont exposés dans le tableau 6.

\section{TABLEAU 6}

L'indice WPN (WPNI) du lait de consommation (valeurs de transmission, $420 \mathrm{~nm}$ )

\begin{tabular}{ll|c|c|c|c}
\hline \multicolumn{2}{c|}{ Traitement thermique } & Moyenne & Minimale & Maximale & Déviat. stand. \\
\cline { 1 - 2 } & & & & & \\
Pasteurisation & $(\mathrm{n}=5)$ & 54,6 & 50,6 & 59,1 & 2,9 \\
UHT, direct & $(\mathrm{n}=6)$ & 71,4 & 69,7 & 73,2 & 3,2 \\
UHT, indirect & $(\mathrm{n}=9)$ & 85,2 & 81,0 & 88,3 & 4,4 \\
Stérilisation en PE & $(\mathrm{n}=5)$ & 94,2 & 90,5 & 97,3 & 2,7 \\
Stérilisation en verre & $(\mathrm{n}=5)$ & 94,8 & 92,6 & 98,6 & 2,1 \\
& & & & & \\
\hline
\end{tabular}

$\mathrm{n}=$ nombre d'échantillons.

Pour le lait pasteurisé, nous avons mesuré un indice WPN moyen de 54,6 contre 71,4 pour le lait UHT à réchauffement direct et 85,2 pour le lait UHT à réchauffement indirect. Certains laits UHT à réchauffement indirect avaient une valeur WPNI comparable à celle du lait stérilisé dans une bouteille de polyéthylène (environ 90). En moyenne, la méthode WPNI a permis de bien faire la distinction entre les différentes catégories de lait de consommation examinées.

\section{III.3.2. Degré de turbidité}

Le test d'Aschaffenburg est généralement employé pour juger un lait de consommation et consiste en l'observation visuelle de l'apparition d'un trouble. Ce mode opératoire est entaché de subjecti- 
vité, certainement dans les cas-limites. C'est pourquoi nous avons recherché si la détermination néphélométrique de la turbidité ne peut pas servir de critère d'intensité de réchauffement et permettre ainsi de distinguer les différents types de lait de consommation.

Le tableau 7 donne un aperçu du degré de turbidité (NTU) des laits de consommation examinés. Les résultats montrent que l'on a

\section{TABLEAU 7}

La turbidité (NTU) du lait de consommation

\begin{tabular}{ll|c|c|c|c}
\hline \multicolumn{1}{c|}{ Traitement thermique } & Moyenne & Minimale & Maximale & Déviat. stand. \\
\hline & & & & & \\
& & & & \\
Pasteurisation & $(\mathrm{n}=8)$ & 771 & 645 & 895 & 29,3 \\
UHT, direct & $(\mathrm{n}=11)$ & 181 & 86 & 330 & 51,8 \\
UHT, indirect & $(\mathrm{n}=19)$ & 14,2 & 3,0 & 44,5 & 12,1 \\
Stérilisation en PE & $(\mathrm{n}=16)$ & 1,4 & 0,5 & 5,9 & 1,6 \\
Stérilisation en verre & $(\mathrm{n}=9)$ & 0,8 & 0,5 & 1,3 & 0,3 \\
\end{tabular}

$\mathrm{n}=$ nombre d'échantillons.

relevé, en moyenne, d'importantes différences en turbidité, mesurée au néphélomètre, entre les différents types de lait de consommation. La turbidité moyenne du lait pasteurisé était de 771 NTU et notablement plus élevée que celle du lait UHT. Après un traitement UHT direct, le degré de turbidité moyen était de 181 NTU, contre 14 NTU après un traitement indirect. Le lait stérilisé dans la bouteille avait une turbidité moyenne d'environ 1. Dans quelques cas exceptionnels, les valeurs relevées pour le lait stérilisé dans la bouteille et pour le lait UHT chauffé indirectement se chevauchaient. En moyenne, on relevait une nette différence entre les différents types de lait de consommation.

Les essais ont démontré qu'un degré de turbidité moyen devient visuellement perceptible à partir de 20 NTU.

\section{III.3.3. Teneur en hydroxyméthylfurfural}

L'hydroxyméthylfurfural est un produit intermédiaire de la réaction de Maillard. Sa formation est fortement dépendante de la tem- 
pérature. Les taux d'HMF que nous avons relevés dans le lait de consommation sont reproduits dans le tableau 8.

La distinction entre les teneurs en HMF des différents types de lait de consommation était, en moyenne, bien évidente. Le lait pasteurisé avait une teneur moyenne de 2,4 $\mu \mathrm{mol} / \mathrm{l}$. Dans le cas des laits UHT, on pouvait distinguer le produit du réchauffement direct $(5,3 \mu$ $\mathrm{mol} / \mathrm{l})$ de celui du réchauffement indirect $(10,0 \mu \mathrm{mol} / \mathrm{l})$. La teneur moyenne du lait stérilisé dans la bouteille par la méthode classique était supérieure à $17 \mu \mathrm{mol} / 1$.

\section{TABLEAU 8}

La concentration en HMF du lait de consommation ( $\mu$ mol/l)

\begin{tabular}{lr|c|c|c|c}
\hline \multicolumn{2}{c|}{ Traitement thermique } & Moyenne & Minimale & Maximale & Déviat. stand. \\
& & & & & \\
Pasteurisation & $(\mathrm{n}=7)$ & 2,4 & 0,5 & 4,9 & 0,7 \\
UHT, direct & $(\mathrm{n}=11)$ & 5,3 & 3,1 & 7,4 & 1,5 \\
UHT, indirect & $(\mathrm{n}=15)$ & 10,0 & 5,2 & 16,8 & 2,7 \\
Stérilisation en PE & $(\mathrm{n}=12)$ & 17,5 & 11,7 & 23,9 & 3,7 \\
Stérilisation en verre & $(\mathrm{n}=9)$ & 19,1 & 12,9 & 24,1 & 3,9 \\
& & & & & \\
\hline
\end{tabular}

$\mathrm{n}=$ nombre d'échantillons.

Nous avons toutefois constaté un chevauchement important des quantités d'HMF des différentes catégories, ce qui rend la détermination de l'HMF moins sûre comme test de différenciation. De plus, l'HMF est sujet à des réactions de synthèse et de dégradation pendant la conservation et ces réactions sont d'autant plus marquées que la température de conservation est plus élevée (Mottar et al., 1979).

\section{CONCLUSION}

Une étude comparative, organoleptique, nutritionnelle et analytique, a démontré que le lait chauffé à ultra-haute température et soutiré dans des conditions aseptiques se distingue en règle générale 
comme une catégorie particulière du lait pasteurisé et stérilisé par la méthode traditionnelle. La charge thermique exercée dans certaines installations de réchauffement indirect est toutefois si élevée que les caractéristiques du lait ainsi traité ne diffèrent que peu de celles du lait stérilisé dans la bouteille.

Sur le plan technologique, il est possible d'obtenir par un traitement à ultra-haute température un lait qui répond dans une très large mesure aux conditions de qualité exigées par le consommateur. Le réchauffement UHT peut être réalisé, en principe, par deux procédés technologiques différents. Pour chaque procédé considéré séparément, il existe de grandes différences sous le rapport de l'intensité de chauffage. Il faudrait cependant s'efforcer à maintenir dans des limites acceptables l'intensité de chaleur à laquelle le lait est soumis au cours d'un traitement UHT.

Dans certains cas-limites, le test d'Aschaffenburg ne permet pas de faire une distinction visuelle entre le lait UHT et le lait stérilisé dans la bouteille. Le test de turbidité qui consiste à mesurer le degré de turbidité par néphélométrie et l'indice WPN (WPNI) donnent une idée plus exacte du traitement thermique subi. Ces méthodes alternatives permettent une caractérisation objective du lait de consommation.

\section{R és u m é}

Les effets de différents traitements thermiques ont fait l'objet d'une étude comparative, au cours de laquelle on a également recherché s'il était possible de distinguer par des moyens analytiques les différentes catégories de lait de consommation.

Le goût du lait UHT a été jugé moins favorablement que celui du lait fraîchement pasteurisé. La différence était d'autant plus marquée que le réchauffement avait été plus intense.

Les pertes moyennes en lysine disponible, en vitamine $\mathrm{B}_{12}$, en acide folique et en vitamine $\mathrm{C}$ du fait de la pasteurisation étaient faibles. L'effet du réchauffement à ultra-haute température était plus grand. Dans certains cas de traitement indirect, la perte de la valeur nutritive était analogue à celle d'une stérilisation en bouteille.

Le degré de réchauffement moyen du traitement UHT était significativement plus élevé que celui de la pasteurisation et égalait dans certains systèmes indirects celui d'une stérilisation classique.

Le lait UHT se distingue en règle générale comme un type particulier de lait pasteurisé et stérilisé dans la bouteille par la méthode traditionnelle. Toutefois, dans certains cas-limites, le test d'Aschaffenburg ne permet plus de distinguer un lait UHT d'un lait stérilisé dans la bouteille. Dans certains appareils à chauffage indirect, le 
réchauffement est, en effet, si intense que l'on obtient également une réaction négative.

Un test de turbidité modifié, où le degré de turbidité est mesuré par néphélométrie, et l'indice WPN (WPNI) procurent une idée exacte de la charge thermique subie. Ces méthodes alternatives permettent de caractériser objectivement un lait de consommation.

\section{S u $\mathrm{m} \mathbf{m}$ a $r \mathrm{y}$}

Comparative research into the effect of several thermal process on milk was carried out. The possible analytical distinction between the different kinds of market milk was also investigated.

The taste of UHT milk was less favourably judged than that of freshly pasteurized milk. The difference percepted was the greater according as the milk had been heated more intensely.

The mean losses in available lysine, vitamine $B_{12}$, folic acid and vitamine $\mathrm{C}$ following pasteurization were small. Following UHT treatment the mean losses were higher. For particular indirect heating procedures the loss of the nutritive value was comparable to that of traditional in bottle sterilization.

The mean heating level of the UHT treatment was noticeably higher than that of pasteurization for particular indirect heating procedures it was even the same as that of classic sterilization.

As a rule UHT milk distinguishes itself from pasteurized milk and milk sterilized after bottling. In some cases, however, the Aschaffenburg test does not allow distinction between UHT milk and milk sterilized after bottling; with particular indirect plants heating is so intense that the reaction is also negative.

A modified turbidity test, in which the turbidity is measured by nephelometric determination, and the WPN-index seem to be suitable for objective classification of heat-treated milks.

Reçu pour publication en mai 1979.

\section{Références}

Association of official analytical chemists (1975). - Methods of Analysis. A.O.A.C. 12th Edition.

Aschaffenburg (R.) and Drewey (J.) (1959). - New procedure for the routine determination of the various non-casein proteins of milk. 15th Int. Dairy Congres, 3 (5), 1631.

Воютн (V.) (1971). - Determination of FDNB-available lysine. A modified carpenter procedure. J. Sci. Fd. Agric., 22, 658. 
Hansson (E.) et Olsson (H.) (1974). - Recherches sur l'oxydation de l'acide ascorbique dans le lait conditionné sous carton. Rev. Lait. Française, 98, 85.

International Dairy Federation (1972). - Monograph on UHT-milk. Brussels.

KEENEY (M.) and BASSETTE (R.) (1959). - The determination of hydroxymethylfurfural. J. Dairy Sci., 42, 945.

Kuramoto (S.), Jenness (R.), Coulter (S. T.) and Choi (R. P.) (1959). - Standardization of the Harland-Ashworth test for whey protein nitrogen, 42, 28.

MotTaR (J.) and NaudTs (M.) (1979). - Some observations on the differences between UHT milk and in-container sterilized milk. Memo Group B 21, International Dairy Federation.

Mottar (J.), Waes (G.), Moermans (R.) and Naudts (M.) (1979). - Sensoric changes of UHT milk during uncouled storage. Milchwiss. (in press). 